\title{
First detection of vibrationally excited water masers toward embedded Young Stellar Objects
}

\author{
Tarja Liljeström \\ Helsinki University of Technology, Metsähovi Radio Observatory, \\ Otakaari 5.A, FIN-02150 Espoo, Finland \\ Anders Winnberg and Roy Booth \\ Chalmers University of Technology, Onsala Space Observatory, \\ S-43992 Onsala, Sweden
}

\begin{abstract}
We report the first detection of vibrationally excited water maser emission in the circumstellar envelope of two young stellar objects (W51M, Z CMa). The results suggest that the observed $96 \mathrm{GHz}$ water maser emission traces hot gas in an accretional stage of the protostellar evolution.
\end{abstract}

\section{Introduction}

Radio emission from $\nu_{2}$ vibrationally excited water lines at 96,232 , and $658 \mathrm{GHz}$ have previously been reported in a few oxygen-rich late-type stars. The masing para-water line at $96 \mathrm{GHz}$ has been seen only toward the supergiant VY CMa, whereas the para-water line at $232 \mathrm{GHz}$ has been found in VY CMa and W Hya (Menten \& Melnick 1989). On the other hand, the ortho-water line at $658 \mathrm{GHz}$ has been detected in some ten late-type stars (Menten \& Young 1995).

The upper energy levels of the $\nu_{2}$ vibrationally excited water lines at 96 , 232 , and $658 \mathrm{GHz}$ are 3065,3462 , and $2329 \mathrm{~K}$, respectively. The high excitation temperature requirement explains the rarity of $96 \mathrm{GHz}$ maser sources. In the only earlier reported $96 \mathrm{GHz}$ source (VY CMa), the maser emission seems to originate within a few stellar radii from the central star in the hot inner edge of the circumstellar envelope, where sufficient dust has not yet formed to start the outward expansion of the circumstellar envelope (Menten \& Melnick 1989).

Attempts have been made to search for vibrationally excited water masers toward embedded young stellar objects (e.g., Orion KL, W49N, W51M), but with negative results (Menten \& Melnick 1989; Menten \& Young 1995). Here we present the first detection of the vibrationally excited $\left(\nu_{2}=1\right)$ water maser emission (from the rotational transition $440-533$ ) toward the compact concentration of $22 \mathrm{GHz}$ masers near the reference position of W51M, hereafter called the protostellar "cocoon" (Genzel et al. 1978; Leppänen, Liljeström, \& Diamond 1998) and toward the hot, FU Orionis-type accretion disk of Z CMa (Hartmann et al. 1989; Liljeström \& Olofsson 1997). 


\section{Observations}

The $20 \mathrm{~m}$ radio telescope of Onsala Space Observatory was used to search for vibrationally excited water masers at $96.261161 \mathrm{GHz}$ toward massive or intermediate-mass young stellar objects, known either as hot water maser sources (Melnick et al. 1993) or as strongly accreting sources.

At $96 \mathrm{GHz}$ the beam width of the antenna is 39 " and the main beam efficiency 0.57 . The observations were performed under very good weather conditions in a dual beam-switching mode with a 11' throw in azimuth. As back ends we used three spectrometers with channel widths of $0.04,0.16$, and 0.78 $\mathrm{km} \mathrm{s}^{-1}$. The reason for using multiple spectrometers with different resolutions was to verify possible detections of the expected very weak $96 \mathrm{GHz}$ water line.

The data were calibrated with standard chopper-wheel technique. The spectra are presented in units of the main beam brightness temperature, $\mathrm{T}_{M B}$. A brightness temperature of $1 \mathrm{~K}$ corresponds to a flux density of $6.7 \mathrm{Jy}$. The Doppler velocities in this study refer to the Local Standard of Rest (LSR).

\section{Results and Discussion}

\subsection{W51M}

Figure 1 (left) displays a clear detection of the vibrationally excited water line at $96 \mathrm{GHz}$ toward the protostellar maser cocoon of W51M (Genzel et al. 1978; Leppänen et al. 1998). A Gaussian fit to the high-resolution (channel width 0.04 $\mathrm{km} \mathrm{s}^{-1}$ ) spectrum of W51M yields a peak brightness temperature of $0.4 \mathrm{~K}$ at a Doppler velocity of $63.4 \mathrm{~km} \mathrm{~s}^{-1}$ and a line width (FWHM) of $0.4 \mathrm{~km} \mathrm{~s}^{-1}$. The lower-resolution spectrum of W51M (channel width $0.16 \mathrm{~km} \mathrm{~s}^{-1}$ ) yields $\mathrm{T}_{M B}$ $=0.17 \mathrm{~K}, \mathrm{~V}_{L S R}=63.2 \mathrm{~km} \mathrm{~s}^{-1}$, and a line width of $0.7 \mathrm{~km} \mathrm{~s}^{-1}$. The signalto-noise ratio is 3.4 and 7 in the high-resolution and low-resolution spectrum, respectively. The detected line signals correspond to $1-3 \mathrm{Jy}$.

It is noteworthy that the $96 \mathrm{GHz}$ water line is discovered in W51M, which differs from other $22 \mathrm{GHz}$ maser sources in the sense that it does not show the common bipolar maser outflow. This suggests that the $96 \mathrm{GHz}$ line traces an earlier stage of stellar evolution, where accretion from the protostellar cocoon onto the protostar is still important, in accordance with $22 \mathrm{GHz}$ proper motion results of W51M (Genzel et al. 1981). In support of this we note that the line velocity at $96 \mathrm{GHz}$ is redshifted by some $1.9 \mathrm{~km} \mathrm{~s}^{-1}$ as compared to the velocity centroid of the compact $22 \mathrm{GHz}$ maser cocoon of W51M (Leppänen et al. 1998). Moreover, the flux densities of the low-velocity $22 \mathrm{GHz}$ masers of W51M (Leppänen et al. 1998) agree with theoretical predictions of collapsing protostellar envelopes (Ceccarelli, Hollenbach, \& Tielens 1996).

\subsection{Z Canis Majoris}

Figure 1 (right) displays the observed $96 \mathrm{GHz}$ water line toward the FU Orionistype object Z CMa, for which infall motions have been reported (Hartmann et al. 1989; Liljeström \& Olofsson 1997). The high-resolution spectrum (channel width $0.16 \mathrm{~km} \mathrm{~s}^{-1}$ ) of $\mathrm{Z} \mathrm{CMa}$ has a peak brightness temperature of $0.44 \mathrm{~K}$ (corresponding to $2.9 \mathrm{Jy}$ ) at a Doppler velocity of $13.9 \mathrm{~km} \mathrm{~s}^{-1}$ and a line width 
of $0.60 \mathrm{~km} \mathrm{~s}^{-1}$. The low-resolution spectrum (channel width $0.78 \mathrm{~km} \mathrm{~s}^{-1}$ ) has a peak brightness temperature of $0.14 \mathrm{~K}$ (corresponding to $0.94 \mathrm{Jy}$ ) at a Doppler velocity of $14.0 \mathrm{~km} \mathrm{~s}^{-1}$. The signal-to-noise ratio is 4.1 and 3.6 in the highresolution and low-resolution spectrum, respectively.

The $96 \mathrm{GHz}$ line seen toward $\mathrm{Z} \mathrm{CMa}$ is not as convincing as in W51M. However, it is in accordance with $2 \mu \mathrm{m}$ vibration-rotation water band observations of Z CMa (Liljeström \& Olofsson 1997), which indicate a kinetic temperature about $2000 \mathrm{~K}$ in the inner disk region of $\mathrm{Z} \mathrm{CMa}$. The heating probably follows from mass accretion through the disk onto the protostar. However, the hot water vapor traced by the $96 \mathrm{GHz}$ emission does not take part in infalling motions, because the line velocity is not redshifted. Therefore, other heating processes may be active, as argued in the following.

Interferometric continuum observations of $\mathrm{Z} \mathrm{CMa}$ at $110 \mathrm{GHz}$ (Liljeström \& Bergman 1996) reveal that the disk of $\mathrm{Z} C \mathrm{CMa}$ is very massive $\left(1.5 \mathrm{M}_{\odot}\right)$ and shows a one-armed trailing spiral feature. Therefore, non-axisymmetric gravitational instabilities are probably present, transporting disk material inward and angular momentum outward manifested as trailing spiral features (Shu et al. 1990). The heating required for the observed $96 \mathrm{GHz}$ water maser emission in $\mathrm{Z} \mathrm{CMa}$ may thus result from nonviscous dissipation of a spiral density wave (Shu et al. 1993). Furthermore, because gravitational instabilities drive disk accretion by first building up the mass in the disk and then dumping it sporadically onto the protostar, they naturally explain the FU Orionis-type outbursts of Z CMa.

\section{References}

Ceccarelli, C., Hollenbach, D., \& Tielens, A. 1996, ApJ, 471, 400

Genzel, R., et al. 1978, A\&A, 66, 13

Genzel, R., et al. 1981, ApJ, 247, 1039

Hartmann, L., et al. 1989, ApJ, 338, 1001

Leppänen, K., Liljeström, T., \& Diamond, P. 1998, ApJ, 507, 909

Liljeström, T., \& Bergman, P. 1996, in URSI/IEEE/IRC XXI Convention on Radio Science, ed. P. Piironen \& A. Räisänen (Espoo: Helsinki University of Technology), S 222, 37

Liljeström, T., \& Olofsson, G. 1997, ApJ, 478, 381

Melnick, G., Menten, K., Phillips, T., \& Hunter, T. 1993, ApJ, 416, L37

Menten, K., \& Melnick, G. 1989, ApJ, 341, L91

Menten, K., \& Young, K. 1995, ApJ, 450, L67

Shu, F., et al. 1990, ApJ, 358, 495

Shu, F., et al. 1993, in Protostars and Planets III, ed. E. H. Levy \& S. Mathews (Tucson: Univ. Arizona Press), 3 

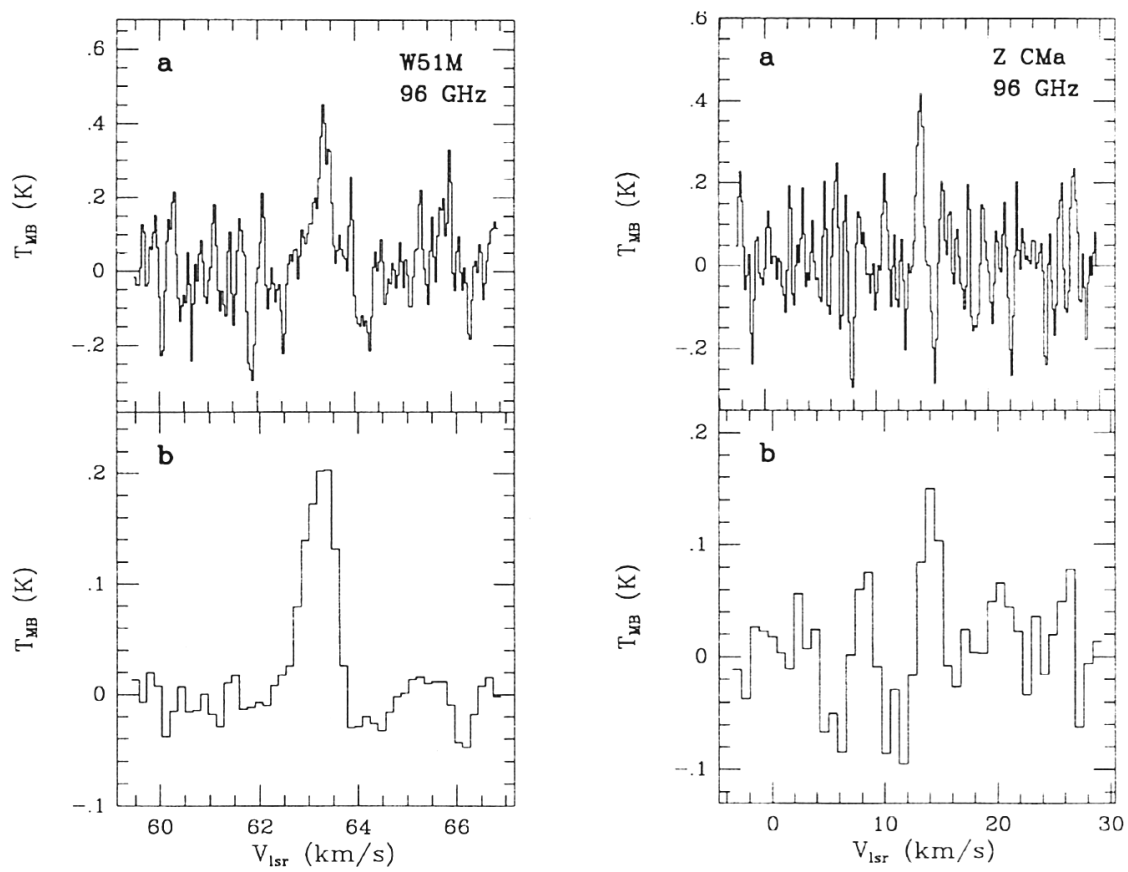

Figure 1. $\quad 96 \mathrm{GHz}$ water maser spectra toward W51M (left) and Z CMa (right); (a) high-resolution spectrum, (b) low-resolution spectrum.

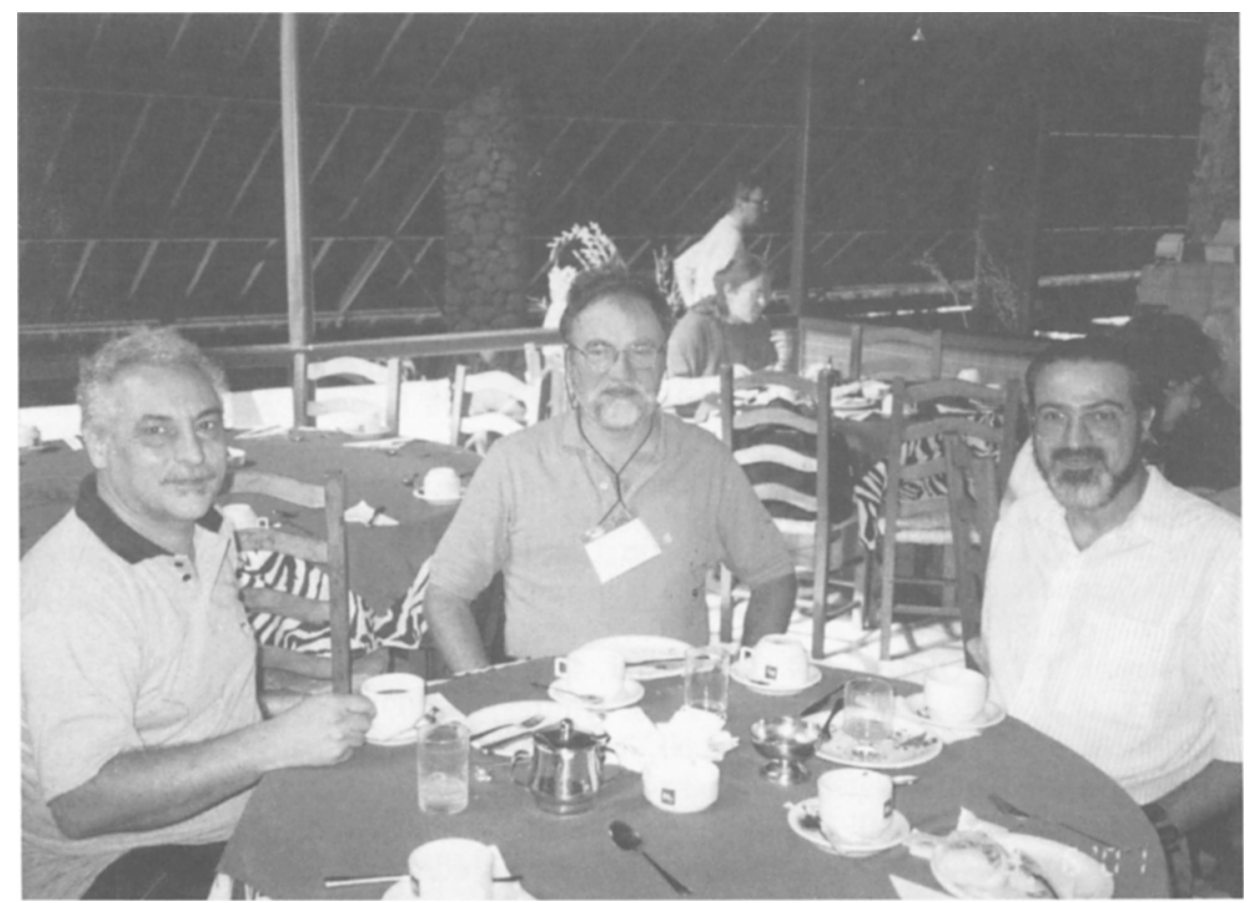

